\title{
Recurrent intraductal papillary mucinous neoplasms of the pancreas mimicking ampullary cancer
}

\author{
Masataka Hayashia, Kenjiro Datea, Tomoko Kodamab ${ }^{\mathrm{b}}$, Akihiro Yamasuji ${ }^{\mathrm{b}}$, Kou Fukumorib, Koji Tamuraa,c, \\ Naoki Maehara ${ }^{a}$
}

Fujimoto General Hospital, Miyazaki; Hamanomachi Hospital, Fukuoka, Japan

\begin{abstract}
Although several studies have reported that some patients developed metachronous/recurrent intraductal papillary mucinous neoplasms (IPMNs) after partial pancreatectomy, recurrence of IPMN mimicking ampullary cancer is extremely rare. We report the case of a 62 -year-old man who developed recurrent IPMN mimicking ampullary cancer. Every 3-6 months, the patient had received surveillance with computed tomography after distal pancreatectomy for IPMN, high-grade, pancreatobiliary type. However, a villous tumor at the major duodenal papilla was found incidentally by upper gastrointestinal endoscopy 2 years and 3 months after initial surgery, and the biopsy result was adenocarcinoma. Endoscopic ultrasonography showed a tumor at the periampullary lesion; however, the origin of the tumor could not be determined definitively. Remnant total pancreatectomy was performed, and the histological diagnosis revealed IPMN, high-grade, pancreatobiliary type. Some patients develop recurrent IPMN mimicking ampullary cancer; thus, careful surveillance for periampullary lesions as well as remnant pancreas should be performed.
\end{abstract}

Keywords Intraductal papillary neoplasm of the pancreas, recurrent, surveillance, endoscopic ultrasonography, ampullary tumor

Ann Gastroenterol 2020; 33 (4): 1-4

\section{Introduction}

Intraductal papillary mucinous neoplasms (IPMNs) of the pancreas are cystic precursor lesions characterized by multiple occurrences in the same pancreas. After partial pancreatectomy for IPMNs, the remnant pancreas still remains at risk for secondary lesions, despite the negative surgical margin [1]. Although several studies have reported that some patients developed metachronous/recurrent IPMNs in the remnant pancreas [2,3], IPMN resembling an ampullary tumor is extremely rare [4], because IPMNs arise from the epithelial cells in the pancreatic ductal systems. In addition, an adequate

Department of a Surgery (Masataka Hayashi, Kenjiro Date, Koji Tamura, Naoki Maehara); ${ }^{b}$ Gastroenterology (Tomoko Kodama, Akihiro Yamasuji, Kou Fukumori), Fujimoto General Hospital, Miyazaki; 'Department of Gastroenterological Surgery, Hamanomachi Hospital, Fukuoka (Koji Tamura), Japan

\section{Conflict of Interest: None}

Received 15 March 2020; accepted 11 May 2020; published online 29 May 2020

Correspondence to: Kenjiro Date, MD, PhD, Department of Surgery, Fujimoto General Hospital, 17-1 Hayasuzu, Miyakonojo, Miyazaki, 885-0055, Japan, e-mail: k-date@surg1.med.kyushu-u.ac.jp

DOI: https://doi.org/10.20524/aog.2020.0501 surveillance protocol after partial pancreatectomy for IPMNs has yet to be established.

Here we report on a rare patient who developed recurrent IPMNs mimicking ampullary cancer, detected not by crosssectional imaging study surveillance, but by an endoscopic procedure.

\section{Case report}

A 59-year-old man was admitted to our hospital for detailed examination of cystic lesions in the pancreas. He had neither a past history nor a family history of pancreatic cancer. Laboratory examination revealed the serum carcinoembryonic antigen and the carbohydrate antigen 19-9 were within normal limits. Enhanced computed tomography (CT) showed a dilated main pancreatic duct with an enhanced mural nodule (30 $\mathrm{mm}$ in diameter) in the pancreas body (Fig. 1A). Magnetic resonance cholangiopancreatography (MRCP) and endoscopic ultrasonography (EUS) showed a $10-\mathrm{mm}$ dilated main pancreatic duct and mural nodule in the body, while the main pancreatic duct in the head was intact (Fig. 1B,C). During the duodenoscopy, the main duodenal papilla was intact (Fig. 1D). The patient had undergone distal pancreatectomy (Fig. 2A,B) based on the imaging findings, suggesting the 
malignant potential of the IPMNs [5]. The histological diagnosis revealed IPMN, high-grade, pancreatobiliary type (Fig. 2C,D). In the immunohistochemical analysis, the tumor cells were positive for MUC1, negative for MUC2 and positive for MUC5AC (Fig. 2E-G), supporting pancreatobiliary type. Intraoperative frozen and permanent section histology of the pancreatic cut margin was negative for carcinoma cells. Fluid collection around the peripancreatic space, presumably due to postoperative pancreatic fistula, had been detected by CT on the 8th postoperative day (data not shown). However, the patient had shown no symptoms and had been discharged on the 24th postoperative day.

Although International consensus guidelines recommend surveillance with alternate CT and MRCP/EUS [1], we had decided to perform postoperative surveillance using CT for
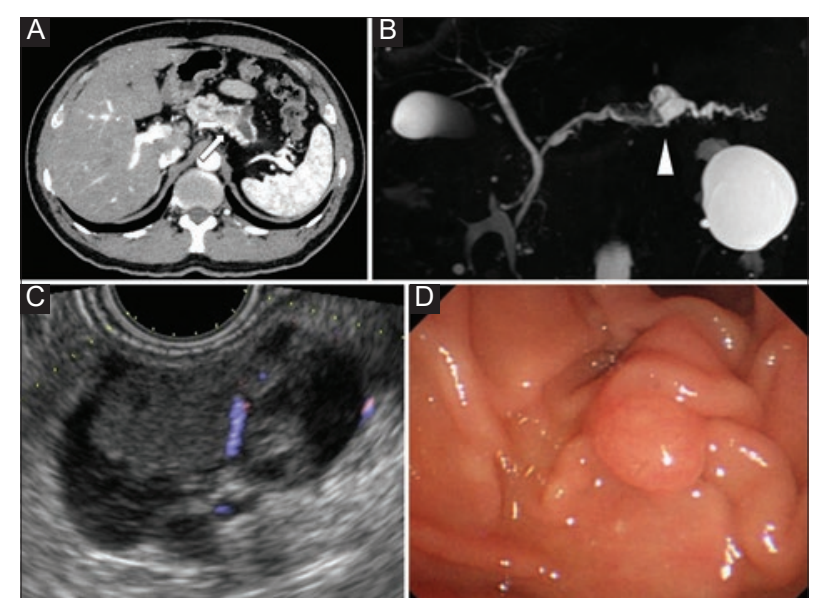

Figure 1 Imaging findings at the initial surgery. (A) Enhanced computed tomography shows a dilated main pancreatic duct with an enhanced mural nodule ( $30 \mathrm{~mm}$ in diameter) in the pancreas body (arrow). (B) Magnetic resonance cholangiopancreatography shows a $10-\mathrm{mm}$ dilated main pancreatic duct and mural nodule in the pancreas body (arrow head), while the main pancreatic duct is intact. (C) Endoscopic ultrasonography shows a dilated main pancreatic duct and mural nodule in the pancreas body, while the main pancreatic duct is intact. (D) Duodenoscopy shows intact main duodenal papilla surveillance of the peripancreatic fluid collection as well as postoperative recurrence. The patient had undergone $\mathrm{CT}$ at one month, and then every 3-6 months after surgery, and the fluid collection had disappeared one year after initial surgery. There were no cystic lesions in the remnant pancreas 1 year after surgery (Fig. 3A). Although the emergence of a cystic lesion (15 $\mathrm{mm}$ in diameter) at the pancreas head was observed one and a half years after surgery, there were no suspicious signs of malignancy (Fig. 3B) and this lesion had not demonstrated any significant changes 2 years after surgery. However, a villous tumor at the duodenal papilla was found incidentally by upper gastrointestinal endoscopy 2 years and 3 months after surgery. Duodenoscopy and biopsy were performed (Fig. 4A), and the result was adenocarcinoma. CT and magnetic resonance imaging also showed a slightly enhanced mass at the periampullary lesion (Fig. 4B,C). EUS showed a low echoic tumor ( $30 \mathrm{~mm}$ in diameter) at the periampullary lesion (Fig. 4D); however, the origin of the tumor could not be determined definitively. The patient underwent remnant total pancreatectomy (Fig. 5A-C). The histological diagnosis revealed IPMN, high-grade, pancreatobiliary type (Fig. 5D). In the immunohistochemical analysis, the tumor cells were positive for MUC1, negative for MUC2 and positive for MUC5AC (Fig. 5D-G). The patient has remained well without a recurrence 22 months after surgery.

\section{Discussion}

IPMNs of the pancreas are cystic precursor lesions characterized by multiple occurrences in the same pancreas. As the number of patients who undergo surveillance after resection for IPMNs has been increasing, the number of reported patients who developed metachronous/recurrent IPMNs in the remnant pancreas after partial pancreatectomy has also been increasing [2,3]. IPMN resembling an ampullary tumor is rare [4]; thus, recurrent IPMN mimicking ampullary cancer is extremely rare. Although it was difficult to diagnose the origin of the tumor definitively in our patient,

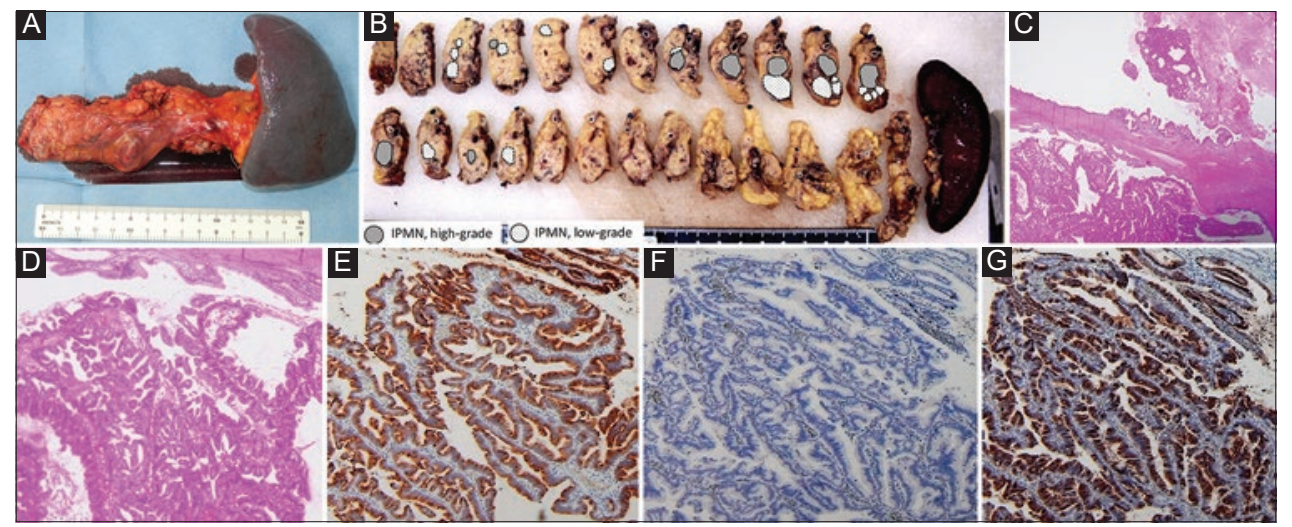

Figure 2 Resected specimen of the initial surgery. (A, B) The tumor located in the pancreas body. (C, D) Microscopic findings of the tumor in the pancreas body (hematoxylin and eosin). Dilated pancreatic ducts are covered with pancreatobiliary type and mucinous epithelium with high-grade dysplasia. In the immunohistochemical analysis, the tumor cells were positive for MUC1 (E), negative for MUC2 (F) and positive for MUC5AC (G) 
the tumor was diagnosed with recurrent IPMNs because of the consistent immunohistochemical findings, as well as

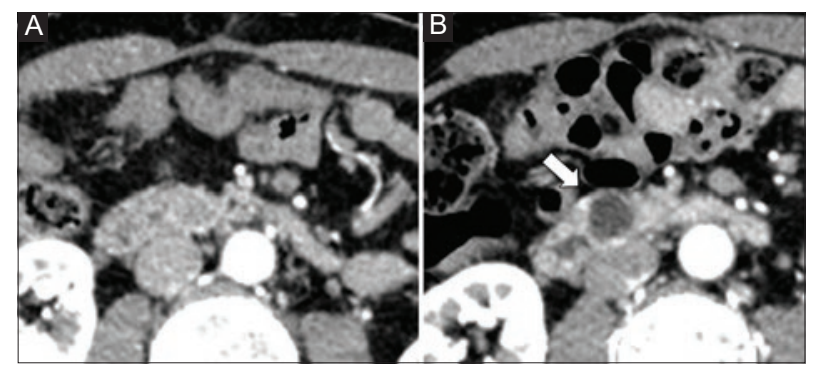

Figure 3 Imaging findings after the initial surgery. (A) Computed tomography shows no cystic lesion in the remnant pancreas one year after surgery. (B) A cystic lesion (15 mm in diameter) appears one and a half years after surgery (arrow)
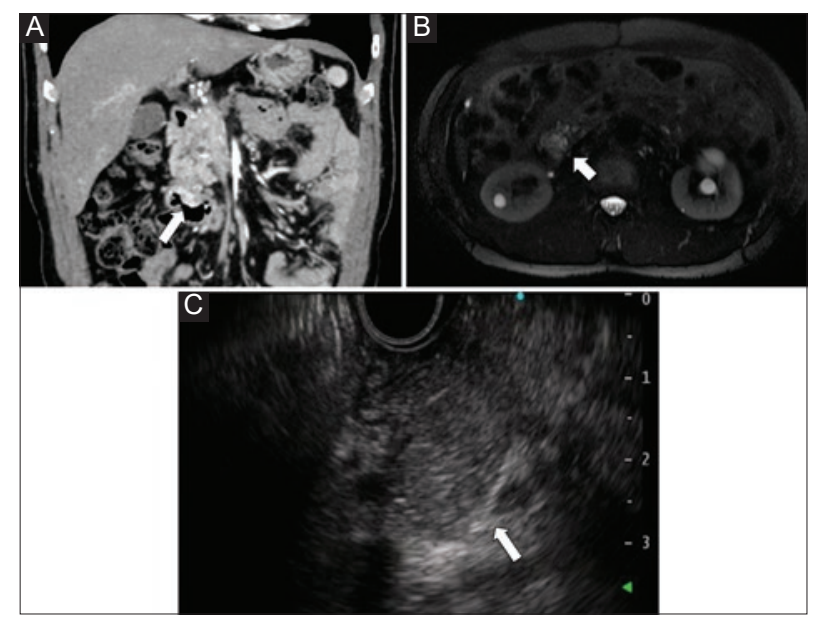

Figure 4 Imaging findings at the second surgery. (A) Duodenoscopy shows an ampullary tumor. (B, C) Computed tomography and magnetic resonance imaging show a slightly enhanced mass at the periampullary lesion without any distant metastasis (arrow). (D) Endoscopic ultrasound shows a low echoic tumor $(30 \mathrm{~mm}$ in diameter) at the periampullary lesion (arrow) the similar morphology shared by the initial and secondary tumors.

There are 3 possible mechanisms underlying the recurrence of IPMNs after partial pancreatectomy for IPMNs: metastatic or local recurrence of carcinoma cells; metachronous multifocal occurrence of IPMNs in the remnant pancreas; and progression of residual IPMNs. In our present patient, 2 metachronous lesions were thought to be discontinuous lesions, because normal epithelium was found at the main pancreatic duct between the 2 distinct lesions. EUS revealed that the main pancreatic duct in the head was intact before the initial surgery, and the histological findings for both lesions were similar; therefore, the second lesion was diagnosed as a skip lesion of the initial lesion, as reported by a group at Kyushu University $[5,6]$.

One of the current issues in the management of IPMNs is establishment of an appropriate surveillance protocol. Kamata et al [7] have recently reported the usefulness of EUS in addition to CT for surveillance of the remnant pancreas after partial pancreatectomy for IPMNs. They have used EUS successfully to detect small lesions, which could not be detected by CT. In our present patient, the emergence of a cystic lesion at the pancreas head was observed one and a half years after surgery. Although there were no suspicious signs of malignancy, the recurrent IPMNs might have been detected if the patient had undergone EUS at that time. Therefore, EUS should be performed if imaging studies show changes in the remnant pancreas.

Several studies have identified the risk factors for developing secondarylesions in the remnant pancreas, high-grade dysplasia in the primary resected IPMNs [8], pancreatobiliary type and the initial tumor being located in the distal pancreas [9]. In our present patient, the histological diagnosis of the initial surgery revealed IPMN, high-grade, pancreatobiliary type, located in the distal pancreas. From this, we should have recognized that our patient was at high risk for developing secondary lesions in the remnant pancreas. Although an adequate surveillance protocol for the remnant pancreas after partial pancreatectomy

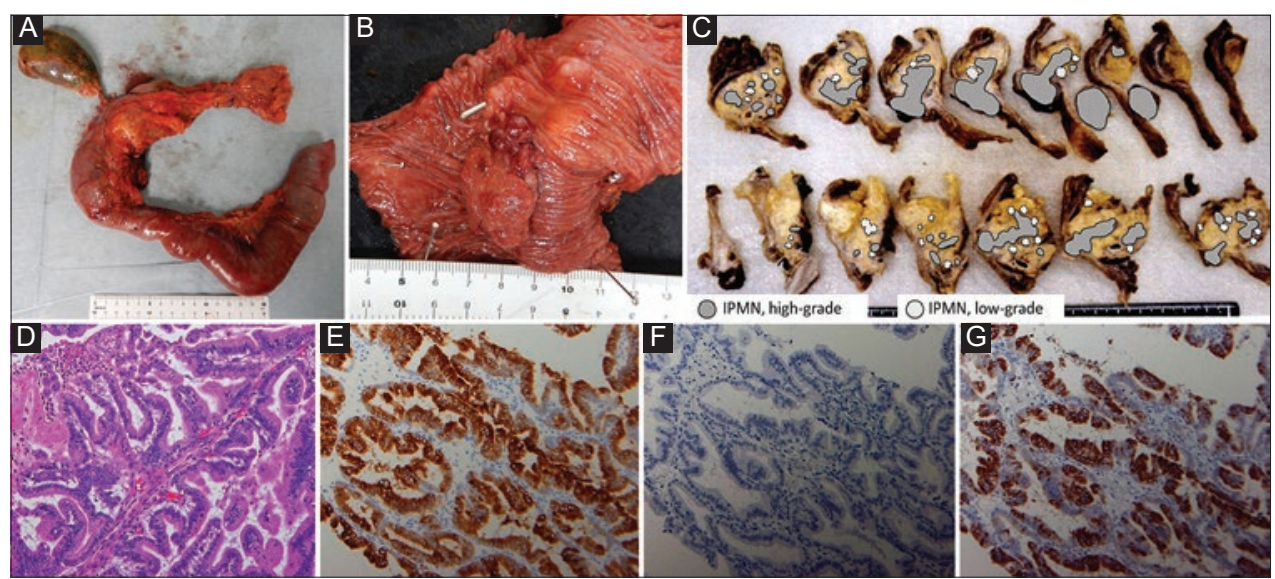

Figure 5 Resected specimen of the second surgery. (A, B) Macroscopic findings of the remnant pancreas. (C) The tumor extends beyond the duodenal ampulla and forms a polypoid mass in the lumen of the duodenum. (D) Microscopic findings of the tumor in the remnant pancreas are similar to those at initial surgery (hematoxylin and eosin). In the immunohistochemical analysis, the tumor cells were positive for MUC1 (E), negative for MUC2 (F) and positive for MUC5AC (G) 
for IPMNs has yet to be established, it might be necessary to add EUS to the surveillance protocol for high-risk patients.

In conclusion, we reported on a patient who developed recurrent IPMNs mimicking ampullary cancer. After partial pancreatectomy, some patients might develop recurrent lesions mimicking ampullary cancer that could be detected, not by cross-imaging, but by endoscopic procedures. EUS should be performed especially when cross-sectional imaging shows some change in the remnant pancreas.

\section{Acknowledgments}

The authors thank Dr. Teruhiko Inoue for making the pathological diagnosis. They also thank Dr Mike Leutchford, MBBS, from Edanz Group (www.edanzediting.com/ac) for editing a draft of this manuscript.

\section{References}

1. Tanaka M, Fernández-Del Castillo C, Kamisawa T, et al. Revisions of international consensus Fukuoka guidelines for the management of IPMN of the pancreas. Pancreatology 2017;17:738-753.
2. He J, Cameron JL, Ahuja N, et al. Is it necessary to follow patients after resection of a benign pancreatic intraductal papillary mucinous neoplasm? J Am Coll Surg 2013;216:657-665.

3. Miller JR, Meyer JE, Waters JA, et al. Outcome of the pancreatic remnant following segmental pancreatectomy for non-invasive intraductal papillary mucinous neoplasm. HPB (Oxford) 2011;13:759-766.

4. Dellaports D, Fragulidis G, Polydorou A, et al. Pancreatic intraductal papillary mucinous neoplasm masquerading as ampullary adenoma: a diagnostic puzzle. Ann Gastroenterol 2020;33:98-100.

5. Tamura K, Ohtsuka T, Matsunaga T, et al. Assessment of clonality of multisegmental main duct intraductal papillary mucinous neoplasms of the pancreas based on GNAS mutation analysis. Surgery 2015;157:277-284.

6. Date K, Ohtsuka T, Fujimoto T, et al. Molecular evidence for monoclonal skip progression in main duct intraductal papillary mucinous neoplasms of the pancreas. Ann Surg 2017;265:969-977.

7. Kamata K, Takenaka M, Minaga K, et al. Value of additional endoscopic ultrasonography for surveillance after surgical removal of intraductal papillary mucinous neoplasms. Dig Endosc 2018;30:659-666.

8. Rezaee N, Barbon C, Zaki A, et al. Intraductal papillary mucinous neoplasm (IPMN) with high-grade dysplasia is a risk factor for the subsequent development of pancreatic ductal adenocarcinoma. HPB (Oxford) 2016;18:236-246.

9. Miyasaka Y, Ohtsuka T, Tamura K, et al. Predictive factors for the metachronous development of high-risk lesions in the remnant pancreas after partial pancreatectomy for intraductal papillary mucinous neoplasm. Ann Surg 2016;263:1180-1187. 